\title{
A Predictive Signature for Oxaliplatin and 5- fluorouracil Based Chemotherapy Inlocally Advanced Gastric Cancer
}

\section{Qinchuan Wang}

Zhejiang University https://orcid.org/0000-0002-2370-6714

Xiyong Liu

City of Hope National Medical Center

Chen Chen

Zhejiang University

Jida Chen

Zhejiang University

\section{Beisi Xu}

Saint Jude Children's Research Hospital

Lini Chen

Zhejiang University

Jichun Zhou

Zhejiang University

Yasheng Huang

Hangzhou Hospital of Traditional Chinese Medicine

\section{Wenjun Chen}

Zhejiang University

\section{Rongyue Teng}

Zhejiang University

Wenhe Zhao

Zhejiang University

Lidan Jin

Zhejiang University

Jun Shen

Zhejiang University

Jianguo Shen

Zhejiang University

Yun Yen ( $\nabla$ y.yenmd@gmail.com )

\section{Linbo Wang}

Zhejiang University 
Research

Keywords: Locally advanced gastric cancer, adjuvant chemotherapy, overall survival, prediction, mutation

Posted Date: June 12th, 2020

DOI: https://doi.org/10.21203/rs.3.rs-34678/v1

License: (c) (i) This work is licensed under a Creative Commons Attribution 4.0 International License.

Read Full License 


\section{Abstract}

Background: Adjuvantchemotherapy(AC)plays a substantial role in the treatment of locally advanced gastric cancer (LAGC), but the response remains poor. Weaims to improve its efficacy in LAGC.

Methods: We identified the expression of eight genes closely associated with platinum and fluorouracil metabolism (RRM1, RRM2, RRM2B, POLH, DUT, TYMS, TYMP, MKI67) in the discovery cohort ( $\mathrm{N}=291)$. And we further validated the findings in TCGA $(\mathrm{N}=279)$ and GEO. Overall survival (OS) was used as an endpoint. Univariate and multivariate Cox models were applied. A multivariate Cox regression model was simulated to predict theOS.

Results: In the discovery cohort,the univariate Coxmodelindicated that AC was beneficial to high-RRM1, high-DUT, low-RRM2, low-RRM2B, low-POLH, low-KI67, low-TYMS or low-TYMP patients, the results were validated in the TCGA cohort. The multivariate Cox model showed consistent results. Cumulative analysis indicated that patients with low C-Score respond poorly to the AC, whereas thehigh and mediumC-Score patients significantly benefit from AC. A risk model based on the above variables successfully predicted the $O S$ in both cohorts (AUC $=0.75$ and 0.67 , respectively). Further validation in a panel of gastric cancer cell (GC) lines ( $\mathrm{N}=37)$ indicated that $\mathrm{C}$-Score is significantly associated with IC50 value to fluorouracil. Mutation profiling showed that C-Score was associated with the number and types of mutations.

Conclusion: In this study, we successfully simulated a predictive signature for the efficacy of AC in LAGC patients and further explored the potential mechanisms. Our findings could promote precision medicine and improve the prognosis of LAGC patients.

\section{Introduction}

Gastric cancer (GC) is the third most common cause of cancer mortality worldwide, killed over 780,000 patients in 2018 (1). For early GC, which is confined to mucosa or submucosa (T1), endoscopic submucosal resection could improve 5 -year survival rate to $84 \%(2,3)$. However, the prognosis of locally advanced GC (LAGC, stage II/III) patients remains poor despite curative surgery, and adjuvant therapies are employed (4). Post-operative chemotherapy has been proved effective; still, many patients showed rapid relapse of the disease and poor overall survival (OS) (5-8). Therefore, how to improve the efficacy of chemotherapy in LAGC patients is a problem urgently needed to be solved.

Multiple strategies have been implemented to enhance the efficacy of chemotherapy, such as neoadjuvant chemotherapy. The MAGIC trial demonstrated that patients received pre-operative ECF (epirubicin, cisplatin, and fluorouracil) chemotherapy showed improved 5-year OS rate (from 23-36\%) compare to postoperative setting (9). FFCD9703 trial improved 5-year OS rate from $24-38 \%$ by combining cisplatin and fluorouracil pre-operatively $(5,10)$. ARTIST trial indicated that post-operative chemoradiation failed to reduce the recurrence rate after curatively resection of GC compared to chemotherapy (11). Notably, ACTS-GC and CLASSIC trials highlight the efficacy of S-1 and capecitabine plus oxaliplatin regimens in improving the 5-year OS rate of post-surgery (R0 resection plus D2 
lymphadenectomy) in stage II/III GC patients $(6,7)$. However, still, over $30 \%$ of LAGC patients showed progressive disease (PD) to chemotherapy, in which mechanisms remain unknown.

Platinum and fluorouracil are the most common reagents in chemotherapy for LAGC (12). It has suggested ribonucleotide reductase (RR), thymidine synthase (TYMS), thymidine phosphorylase (TYMP), deoxyuridine triphosphatase (DUT) and dihydropyrimidine dehydrogenase (DPD) playing substantial roles in the metabolism of 5-Fluorouracil (13). The genetic variants, mRNA, and protein expression of TYMS, TYMP, RR, and DPD were identified as prognostic factors or predictors of chemotherapy response in GC patients (14-19). DNA repair-related genes like excision repair cross-complementing 1 (ERCC1), DNA polymerase $\eta(P O L H)$ were reported to be associated with platinum resistance $(14,20)$. Despite this, resistance to chemotherapy still occurred for lack of valid signature, which eventually results in PD. A 72gene signature of acquired resistance to cisplatin and 5-fluorouracil chemotherapy was identified in 123 metastatic GC patients, which could predict time to progress (21). Another Singaporean study with 318 gastric cancer patients categorized the patients into three subtypes that may be sensitive to different chemo-reagents (22). Among these studies, RR, TYMS, DUT, TYMP, POLH were considered playing key roles in the resistance to cisplatin and 5-fluorouracil (23). However, to date, no consensus has reached in signature for chemotherapy in LAGC.

To identify an optimal signature for chemotherapy in LAGC, we retrospectively enrolled 291 postoperative stage II/III GC patients, and we quantitated the signature of 8 biomarkers (RRM1, RRM2, RRM2B, TYMS, TYMP, DUT, POLH and MKI67) in the fluorouracil and platinum metabolism pathways on slides of patients by immunohistochemistry $(\mathrm{IHC})$. Furthermore, we validated our model on TCGA (The Cancer Genome Atlas, $N=279$ ) and a cell lines microarray ( $N=37)$ from GEO (Gene Expression Omnibus). The findings were further analyzed in mutation profiles of TCGA and GEO datasets, which proposed the possible mechanism. Finally, a multivariable prediction model was addressed in both cohorts to predict the efficacy of fluorouracil and platinum-based chemotherapy for LAGC.

\section{Materials And Methods Ethics Statement}

The protocol of this study was reviewed and approved by the institutional review board (IRB) of Affiliated Sir Run Run Shaw Hospital (SRRSH), Zhejiang University. Written informed consent was obtained from all the patients enrolled in this study.

\section{Study Design}

The study design was depicted in Figure S1. Briefly, we enrolled 472 GC patients who were treated at the department of surgical oncology in Affiliated Sir Run Run Shaw Hospital (SRRSH), Zhejiang University between 1995 and 2011. The inclusion criteria were as followed: 1. Stage II and III Gastric adenocarcinoma with pathology diagnosis, 2 . Inform consented or waiver of consent provided by the 
patient. We excluded patients with the following criteria: 1. Metastatic or Stage I disease; 2. Noneadenocarcinoma or multiple cancer; 3 . No tissue sample obtained; 4 . Failure to provide consent inform; 5. Loss of follow-up after surgery. A total of 291 out of $472 \mathrm{GC}$ patients were finally enrolled in the study. All patients are Han Chinese. Among these patients, 173 of 291 patients had post-surgery adjuvant chemotherapy (Table 1). The combination chemotherapy regimens included folinic acid, 5 -fluorouracil and oxaliplatin (FOLFOX6; 93 Cases); epirubicin, oxaliplatin, and Xeloda (EOX; 13 cases); epirubicin, oxaliplatin and 5-fluorouracil (EOF; 49 cases), multiple chemo-regimen (12 cases), mitomycin and 5fluorouracil (4 cases) and oral S-1 regimen ( 2 cases). All patients were periodically followed up. The TNM stage data for the participants were obtained from the clinical and pathological diagnoses and determined according to the NCCN guidelines for GC (Version 3, 2016) (12). The human tissue samples were obtained from surgery and stored at room temperature after formalin-fixed and paraffin-embedded (FFPE). Correlation result displayed storage time did not affect gene expression in statistical significance $(P>0.05)$. 
Table 1

Host characteristics of SRRSH and TCGA cohorts

\begin{tabular}{|c|c|c|}
\hline Variables/Biomarkers & SRRSH & TCGA \\
\hline †All cases & 291 & 279 \\
\hline \multicolumn{3}{|l|}{ Age } \\
\hline mean(SD) & $59.7(11.6)$ & $65.8(10.6)$ \\
\hline Range & $29-80$ & $29-80$ \\
\hline$<=60$ & 133 & 81 \\
\hline$>60$ & 158 & 193 \\
\hline \multicolumn{3}{|l|}{ Sex } \\
\hline Male & 207 & 179 \\
\hline Female & 84 & 100 \\
\hline \multicolumn{3}{|l|}{ TNM Stage } \\
\hline II & 111 & 117 \\
\hline III & 180 & 162 \\
\hline \multicolumn{3}{|l|}{ *Tumor Grade } \\
\hline low & 35 & 6 \\
\hline medium & 71 & 81 \\
\hline high & 185 & 192 \\
\hline \multicolumn{3}{|l|}{ Histology } \\
\hline Papillary & 4 & NA \\
\hline Mucinous & 24 & NA \\
\hline Signet ring cell & 30 & NA \\
\hline Poor Differentiation & 231 & NA \\
\hline \multicolumn{3}{|l|}{ Tumor location } \\
\hline Proximal & 61 & NA \\
\hline
\end{tabular}

t: Among all biomarkers, there are part of patients' expression information missing: 49 patients in RRM1,48 patients in RRM2, 46 patients in RRM2B, 49 patients in POLH, 47 patients in DUTPASE, 48 patients in KI-67, 46 patients in TYMS and 39 patients in TYMP in SRRSH cohort; In TCGA dataset, age information in 5 cases were missing.

$\ddagger 33$ cases in the SRRSH cohort and 4 cases in the TCGA cohort are undifferentiated, which were included in high tumor grade. 


\begin{tabular}{|c|c|c|}
\hline Variables/Biomarkers & SRRSH & TCGA \\
\hline Body & 63 & NA \\
\hline Distal & 156 & NA \\
\hline Linitis Plastica & 11 & NA \\
\hline \multicolumn{3}{|l|}{ ‡Tumor Size } \\
\hline Yes & 108 & NA \\
\hline No & 174 & NA \\
\hline \multicolumn{3}{|l|}{ Chemotherapy } \\
\hline Yes & 173 & 146 \\
\hline No & 118 & 133 \\
\hline \multicolumn{3}{|c|}{$\begin{array}{l}\text { t: Among all biomarkers, there are part of patients' expression information missing: } 49 \text { patients in } \\
\text { RRM } 1,48 \text { patients in RRM } 2,46 \text { patients in RRM2B, } 49 \text { patients in POLH, } 47 \text { patients in DUTPASE, } 4 \\
\text { patients in KI-67, } 46 \text { patients in TYMS and } 39 \text { patients in TYMP in SRRSH cohort; In TCGA dataset, } \\
\text { age information in } 5 \text { cases were missing. }\end{array}$} \\
\hline \multicolumn{3}{|c|}{$\begin{array}{l}\text { ₹ } 33 \text { cases in the SRRSH cohort and } 4 \text { cases in the TCGA cohort are undifferentiated, which were } \\
\text { included in high tumor grade. }\end{array}$} \\
\hline
\end{tabular}

We validated our results in TCGA (24) and GC cell lines dataset (GSE22183) (22) from GEO. All clinical, pathological information of 279 LAGC cases in 391 GC patients were enrolled in our study (Table 1). In the GSE22183 dataset, $37 \mathrm{GC}$ lines were sequenced with Genome-wide mRNA expression profiles. Among them, 28 gastric cancer cell lines were tested for the $50 \%$ growth inhibition (IC50) to fluorouracil (detailed information was listed in Table S1).

\section{Immunohistochemistry}

The IHC was applied to determine the protein expression levels of biomarkers on FFPE human tissue samples. To normalize the reaction conditions, we reassembled all FFPE tissue samples into multiple tissue arrays as we previously described (15). The accuracy of IHC was validated by quantitative RT-PCR (qRT-PCR) on two parallel samples. Briefly, after deparaffinization, the endogenous peroxidase activity was blocked with $3 \% \mathrm{H}_{2} \mathrm{O}_{2}$ (hydrogen peroxide). The array slides were later incubated with normal goat serum and then applied with a primary antibody. After $\mathrm{H}_{2} \mathrm{O}_{2}$ treatment, the array slides were incubated with horseradish peroxidase-labeled polymer conjugated diaminobenzidine and then counterstained with hematoxylin (DAKO).

An automated imaging system was employed to obtain digital images of the stained sections for subsequent quantitative analyses. Two investigators scored the expression values of biomarkers for each sample in a double-blind manner. Score criteria of RRM1, RRM2, and RRM2B were similar to our previous 
studies $(15,25)$. Both immunoreactivities in the nucleus and cytoplasm were evaluated. Each image was scored based on the following categories: subcellular localization, staining intensity, and/or percentage of stained cells. Based on the intensity of the signal, biomarkers' expressions were classified as negative $(-)$, weakly positive (+), positive (++), or strongly positive (+++) (Figure S2). An expression score of - or + was designated the low expression, and a score of ++ or +++ was classified as the high expression in the following analysis.

\section{Antibodies}

The primary antibodies of RRM1 (1:50), RRM2 (1:10), and RRM2B (1:200) were generated, selected, and tested in our previous experiments $(25,26)$. Thymidine Synthase antibody (1:5, Catalog\#: MBS190051), Ki67 (1:100, Clone: B56), dUTPase (DUT, 1:500, Clone: 1C9), Thymidine Phosphorylase (1:5, Catalog\#: ab180783) and DNA polymerase $\eta$ (1:200, PA5-29063) were purchased from MyBioSource (San Diego, CA), BD Bioscience (San Jose, CA), Novus (Littleton, CO), Abcam (Cambridge, MA) and Thermo Fisher (Waltham, MA), respectively.

\section{Statistics Analysis}

All demographic data, clinicopathological information, and IHC results were coded and entered into the database. Double data entry and logic checks were used for error reduction. SAS 9.4 (SAS Institute, USA), JMP 8.0 Software (SAS Institute, USA) and GraphPad Prism 5.0 (GraphPad Software, USA) were used for the statistical analysis and plotting. Contingency tables and Fisher's exact test were used for the categorical variables to evaluate the association between markers and the response to chemotherapy. The OS was calculated from the date diagnosed to the date of death. Patients who were alive at the last follow-up were censored at that time. The association of each marker with survival and chemotherapy was analyzed using the Kaplan-Meier method and log-rank test. Multivariate analyses were carried out using the Cox proportional hazards model adjust with age, gender, and the interaction effect between chemotherapy and covariates of interest in both the SRRSH cohort and TCGA cohort. All tests were twosided, and $P<0.05$ was considered statistically significant.

\section{Computational Modeling And Verification}

The Cox proportional hazard regression was performed to develop a model to assess and predict the effect of chemotherapy on patients with different expressions of biomarkers adjusting for age, gender, and tumor grade. The proportional hazards assumption was tested, and no violation has been detected among variables of interest. Since the effect of chemotherapy may potentially be influenced by the expression of biomarkers, as well as age, gender and tumor grade, we included interaction terms between chemotherapy (yes/no) and each of the covariates above. The full model indicated that age, RRM1, POLH, TYMS and the interaction terms between chemo and POLH, TYMS and TYMP remained to be 
statistically significantly associated with survival after adjusting for other covariates and interaction effects. To simplify the predictive model, we removed the expression of biomarkers that neither their main effect nor interactive effect has significantly contributed to the model. The Harrell's C-statistic indicated that removing the variables above and terms did not qualitatively decrease the accuracy of the model prediction. To further simplify the model structure, we tried to remove interaction terms between chemo and age, gender and tumor grade, but the prediction accuracy compromised significantly; therefore, we kept those terms within the model. The same model building process has been applied to both SRRSH and TCGA cohort and yielded similar results, however, with a slightly higher Harrell's C-statistic in the SRRSH cohort.

\section{Results}

\section{Expression of 5-Fluorouracil and platinum related markers in LAGC patients who received curative surgery in the SRRSH cohort and TCGA cohort}

We constructed a Multiple Tissue Array (MTA), including all patients enrolled, as we previously reported (15). RRM1, RRM2, RRM2B, DUT, TS, TP, POLH, and Ki67 were stained on the slides of MTAs. All demographic data and contingencies with biomarkers in the SRRSH cohort were listed in Table S2. Among all the GC cases, positive expression of RRM1, RRM2, RRM2B, POLH, DUT, KI67, TS, TP were 110(45.4\%), 135(55.6\%), 137(55.1\%), 154(63.6\%), 140(57.4\%), 155(63.8\%), 141(57.5\%), 59(23.4\%), respectively. All the biomarkers were higher in elderly and male patients except TYMP, compared to younger and female patients. RRM1, DUT, and TYMS were preferentially higher in stage III, low tumor grade and proximal GC patients, whereas RRM2, TYMS and TYMP were associated with poor differentiation.

The baseline data and contingencies of biomarkers of the TCGA cohort were listed in Table S3. All the mRNA expression of selected biomarkers was dichotomized with median levels. In 279 LAGC cases, positive expression of RRM1, RRM2, RRM2B, POLH, DUT, MKI67, TYMS, TYMP were 136(49.0\%), 140(50.0\%), 126(45.0\%), 150(54.0\%), 146(52.0\%), 135(48.0\%), 136(49.0\%), 155(56.0\%), respectively. $P O L H, D U T$ was associated with high tumor grade and younger age.

\section{5-Fluorouracil and platinum related biomarkers predict the efficacy of chemotherapy in LAGC}

Univariate COX Proportional analysis indicated that adjuvant chemotherapy was beneficial to high RRM1 (HR, 0.52, 95\% Cl 0.33-0.84), high DUT (HR,0.65, 95\% Cl 0.43-1.00), low RRM2 (HR,0.51, 95\% $\mathrm{Cl} 0.30-0.84)$, low RRM2B (HR, 0.57, 95\% Cl 0.35-0.97), low POLH (HR,0.34, 95\% Cl 0.19-0.60), low Kl67 (HR,0.52, 95\%Cl 0.29-0.92), low TYMS $(\mathrm{HR}, 0.55,95 \% \mathrm{Cl} 0.31-1.01)$ or low TYMP $(\mathrm{HR}, 0.60,95 \% \mathrm{Cl} 0.40-0.89)$ LAGC patients (Table 2, Fig. 1). To avoid the confounding effect, we further conducted a multivariate Cox proportional analysis. In low RRM2B, low RRM2, low POLH, low KI67 or low RRM1 patients, chemotherapy remained to protect factors in LAGC $(P<0.05)$, whereas other biomarkers showed a consistent trend with the univariate model. 
Table 2

Univariable and Multi-Variable Cox proportional hazard analysis in SRRSH cohort

\begin{tabular}{|c|c|c|c|c|c|}
\hline & & \multicolumn{2}{|l|}{ Univariable Model } & \multicolumn{2}{|c|}{ Multi-Variable Model } \\
\hline Gene signatures & Treatment & $\mathrm{HR}(95 \% \mathrm{Cl})$ & $P$ & tHR (95\%Cl) & $P$ \\
\hline \multicolumn{6}{|l|}{ RRM1 } \\
\hline \multirow[t]{2}{*}{ low } & Surgery alone & Reference & & Reference & \\
\hline & Surgery + AC & $0.74(0.45-1.23)$ & 0.25 & $0.72(0.42-1.23)$ & 0.23 \\
\hline \multirow[t]{2}{*}{ high } & Surgery alone & Reference & & Reference & \\
\hline & Surgery + AC & $0.52(0.33-0.84)$ & 0.007 & $0.66(0.40-1.10)$ & 0.11 \\
\hline \multicolumn{6}{|l|}{ RRM2B } \\
\hline \multirow[t]{2}{*}{ low } & Surgery alone & Reference & & Reference & \\
\hline & Surgery + AC & $0.57(0.35-0.97)$ & 0.04 & $0.55(0.31-0.99)$ & 0.045 \\
\hline \multirow[t]{2}{*}{ high } & Surgery alone & Reference & & Reference & \\
\hline & Surgery + AC & $0.70(0.45-1.09)$ & 0.12 & $0.85(0.72-1.92)$ & 0.51 \\
\hline \multicolumn{6}{|l|}{ RRM2 } \\
\hline \multirow[t]{2}{*}{ low } & Surgery alone & Reference & & Reference & \\
\hline & Surgery + AC & $0.51(0.30-0.84)$ & 0.008 & $0.54(0.32-0.89)$ & 0.02 \\
\hline \multirow[t]{2}{*}{ high } & Surgery alone & Reference & & Reference & \\
\hline & Surgery + AC & $0.78(0.49-1.24)$ & 0.3 & $0.97(0.57-1.66)$ & 0.92 \\
\hline \multicolumn{6}{|l|}{ POLH } \\
\hline \multirow[t]{2}{*}{ low } & Surgery alone & Reference & & Reference & \\
\hline & Surgery + AC & $0.34(0.19-0.60)$ & 0.0003 & $0.37(0.20-0.68)$ & 0.001 \\
\hline \multirow[t]{2}{*}{ high } & Surgery alone & Reference & & Reference & \\
\hline & Surgery + AC & $0.92(0.60-1.42)$ & 0.71 & $1.05(0.66-1.67)$ & 0.84 \\
\hline \multicolumn{6}{|l|}{ dUTPase (DUT) } \\
\hline \multirow[t]{2}{*}{ low } & Surgery alone & Reference & & Reference & \\
\hline & Surgery + AC & $0.63(0.35-1.11)$ & 0.11 & $0.63(0.35-1.31)$ & 0.13 \\
\hline \multirow[t]{2}{*}{ high } & Surgery alone & Reference & & Reference & \\
\hline & Surgery + AC & $0.65(0.43-1.00)$ & 0.05 & $0.82(0.51-1.31)$ & 0.41 \\
\hline
\end{tabular}


Ki67

$\begin{array}{llllll}\text { low } & \text { Surgery alone } & \text { Reference } & & \text { Reference } & \\ & \text { Surgery + AC } & 0.52(0.29-0.92) & 0.02 & 0.49(0.25-0.95) & 0.034 \\ \text { high } & \text { Surgery alone } & \text { Reference } & & \text { Reference } & \\ & \text { Surgery + AC } & 0.74(0.48-1.13) & 0.17 & 0.85(0.55-1.33) & 0.48\end{array}$

TYMS

\begin{tabular}{|c|c|c|c|c|c|}
\hline low & Surgery alone & Reference & & Reference & \\
\hline & Surgery + AC & $0.55(0.31-1.01)$ & 0.05 & $0.60(0.37-1.14)$ & 0.12 \\
\hline high & Surgery alone & Reference & & Reference & \\
\hline & Surgery + AC & $0.78(0.52-1.18)$ & 0.25 & $0.90(0.57-1.42)$ & 0.65 \\
\hline TYMF & & & & & \\
\hline low & Surgery alone & Reference & & Reference & \\
\hline & Surgery + AC & $0.60(0.40-0.89)$ & 0.01 & $0.67(0.44-1.03)$ & 0.07 \\
\hline high & Surgery alone & Reference & & Reference & \\
\hline & Surgery + AC & $0.84(0.45-1.57)$ & 0.6 & $0.97(0.47-1.97)$ & 0.92 \\
\hline
\end{tabular}

Chemo-signature

\begin{tabular}{|c|c|c|c|c|c|}
\hline low & Surgery alone & Reference & & Reference & \\
\hline & Surgery + AC & $1.37(0.65-2.97)$ & 0.41 & $2.11(0.84-5.56)$ & 0.11 \\
\hline medium & Surgery alone & Reference & & Reference & \\
\hline & Surgery + AC & $0.61(0.37-1.00)$ & 0.05 & $0.64(0.38-1.10)$ & 0.1 \\
\hline high & Surgery alone & Reference & & Reference & \\
\hline & Surgery + AC & $0.39(0.21-0.72)$ & 0.003 & $0.44(0.23-0.83)$ & 0.01 \\
\hline
\end{tabular}

The result was validated in the TCGA cohort. Univariate COX model showed high $R R M 1(\mathrm{HR}, 0.47,95 \% \mathrm{Cl}$ 0.24-0.90), low RRM2 (HR, $0.42,95 \% \mathrm{Cl} 0.23-0.76)$, low $R R M 2 B(\mathrm{HR}, 0.38,95 \% \mathrm{Cl} 0.20-0.69)$, low $P O L H$ (HR,0.34, 95\% Cl 0.19-0.60), low MKI67 (HR, 0.40, 95\% Cl 0.21-0.72), low TYMS (HR, 0.36, 95\% Cl 0.190.66) or low $T Y M P(\mathrm{HR}, 0.35,95 \% \mathrm{Cl} 0.16-0.69)$ LAGC patients, high DUT was marginally associated with chemotherapy efficacy $(P=0.05)$, whereas low DUT demonstrated high responsiveness to chemotherapy 
$(P=2.0 \mathrm{E}-04)$. The multivariate Cox model also showed consistent results with the univariate model (Table S4).

\section{C-Score is an ideal predictor for adjuvant therapy efficacy in LAGC}

Cumulative analyses were conducted based on the risk scores (C-Score) generated from the identified candidate biomarkers that were significantly associated with chemotherapy efficacy. The C-Score was calculated as the sum of dichotomized biomarkers (0/1), the value of RRM1 and DUT were labeled as positive for their expressions associating with high efficacy, whereas the rest biomarkers were labeled as a minus for low efficacy. The formula was as following: C-Score = RRM1 + DUT-RRM2-RRM2B-POLHMKI67-TYMS-TYMP. The sum was further categorized as three groups, score -4 and -5 were group low, score -2 and -3 were group medium, score $-1,0,1$ were group high. The univariate COX model indicated that high group response to chemotherapy better than group medium ( $\mathrm{HR}, 0.39,95 \% \mathrm{Cl} 0.21-0.72 \mathrm{vs}$. HR, $0.61,95 \% \mathrm{Cl} 0.37-1.00)$, but the group low had an inadequate response to chemotherapy (HR, $1.37,95 \% \mathrm{Cl}$ 0.65-2.97). The multivariate Cox model indicated similar results (Table 2). Kaplan-Meier analysis showed that patients who received chemotherapy had significantly better OS than patients who received surgery only in group high and group medium (log-rank $P=0.003$, and 0.05 , respectively, Fig. 2 ). The validation in the TCGA cohort also showed similar results that patients in group high and medium response to chemotherapy better than patients who only received surgery $(\mathrm{HR}, 0.36,95 \% \mathrm{Cl} 0.15-0.75, \mathrm{HR}$, $0.47,95 \% \mathrm{Cl} 0.25-0.84$, respectively). The patients in group low demonstrated no difference in death risk and OS in surgery plus chemotherapy and surgery alone GC patients.

To further explore the value of C-Score, we developed a predictive model by including interaction terms between chemotherapy and each of the other covariates. The concordance test indicated that including the interaction effect has significantly improved the prediction accuracy. After performing model selection logic, the final model included age, gender, chemo, tumor grade, RRM1, POLH, TYMD, TYMP, and interaction terms between chemo and each of the other covariates. The model accuracy based on the SRRSH cohort (Harrel's C $=0.7212$ ) was slightly higher compared to that of the TCGA cohort (Harrel's C 0.6823). Also, due to the limited number of observations with survival time longer than three years in the TCGA cohort, here we only reported the model parameters estimated based on the SRRSH cohort. The ROC curves and AUC at specific time points have been presented in Fig. 3A. The AUC fluctuated between 0.75 to 0.81 across different survival tie points. Due to the limited number of observations with survival time longer than 84 months, the ROC value remained the same after the 84th month measurement. The time-dependent AUC and corresponding 95\% confidence limits were presented in Fig. 3B, which indicated that the model prediction accuracy was comparatively stable before the 84th month. Therefore, we suggest applying this model for estimation of no-longer than 5 to 6 -year survival outcomes.

Based on this model, we developed a calculator to predict the hazard ratio of receiving chemotherapy against not receiving chemotherapy for individual patients with different characteristics and biomarker profiles. A sample calculator is available in the supplementary materials in the excel spreadsheet format. The information needed to calculate the HR(chemo/non-chemo) includes gender (0-male/1-female), age 
in year, grade (1 to 4), RRM1, POLH, TYMS, and TYMP (0-no/1-yes) (Attached file1). One limitation of this calculator is that the prediction for low-grade patients may not be applicable. Since chemotherapy has a very significant protective effect for most low-grade patients, which could have potentially masked the modification effect from biomarker profile differences, our predictive model cannot detect the difference due to the biomarker profile variance.

\section{The C-Score is associated with mutation type and burden in LAGC}

To identify the possible mechanism underlying the predictive efficacy of C-Score, we analyzed the mutation profiles of the LAGC from the TCGA cohort. The mutation profiles in group low demonstrated a significantly higher rate of functional mutations than group median and high group (Fig. 4). Notably, the mutation type in low group cases was mainly frame shift ins/del, non-sense, and splice site, which could distinctly alter the protein translating, whereas the primary mutation type in group median and high was missense mutation. The highest frequency of the mutated gene in group low was $A R I D 1 A$, and in group median and high was TP53.

To further validate our findings, we analyzed the association between C-Score biomarkers' expression and IC50 value to 5-fluorouracil in GC cell lines from a published study (22). The result demonstrated that the IC50 value was significantly correlated with chemo-signature in GC cell lines ( $P=0.044$, Table 3$)$.

Moreover, the mutation profile showed a similar distribution pattern to the data from the TCGA cohort, which also showed that high frequency of frameshift mutations in group low and high frequency of missense mutation in group median and group high. The highest mutated gene was EP300 in group low and TP53 in group median and high (Fig. 4).

Table 3

Correlation analysis between C-Score and IC50 in $28 \mathrm{GC}$ cell lines

\begin{tabular}{|lllll|}
\hline IC50/C-Score & $\mathbf{0}$ & $\mathbf{1}$ & $\mathbf{2}$ & \\
\hline Resistant & $2(100 \%)$ & $10(58.8 \%)$ & $2(22.2 \%)$ & \\
\hline Sensitive & $0(0 \%)$ & $7(41.2 \%)$ & $7(77.8 \%)$ & $* P=0.044$ \\
\hline *Chi-square test & & & & \\
\hline
\end{tabular}

\section{Discussion}

In this study, we identified an optimal signature for LAGC in the SRRSH cohort and further validated the results in the TCGA cohort. Eight biomarkers closely correlated with oxaliplatin and 5-fluorouracil metabolisms were detected in 291 LAGC patients in the SRRSH cohort and further validated in 279 LAGC patients in the TCGA cohort. Univariate COX model indicated that adjuvant chemotherapy was beneficial in high RRM1, high DUT, low RRM2, low RRM2B, low POLH, low KI67, low TYMS or low TYMP LAGC patients in both SRRSH and TCGA cohorts. In the multivariable COX model, all the biomarkers demonstrated similar results except DUT in the TCGA cohort, which only showed a consistent trend. C- 
Score based on these biomarkers indicated that patients with high C-Score responded to chemotherapy better than the patients with medium and low C-Score in both cohorts. A Cox regression model (Harrel's C) including age, gender, chemotherapy (yes/no), tumor grade and eight biomarkers was employed to predict the OS of LAGC in both cohorts, which showed that the AUC of SRRSH cohort was 0.7212 , and the AUC of TCGA cohort was 0.6823 . A calculator based on our findings was developed to predict the benefit of chemotherapy. We found that the C-Score of GC cell lines were significantly associated with an IC50 value of 5-fluorouracil, the GC cell lines with low $\mathrm{C}$-Score had significant higher functional mutation rate compare to median and high C-Score, which suggested potential mechanisms. Further, a Calculator was established based on C-Score and other clinic-pathological factors, which could successfully predict OS of LAGC. Thus, we propose that C-Score could be a predictive factor in assisting stratification and treatment decisions of LAGC patients.

The prediction model for chemotherapy efficacy remains controversial in LAGC. A recently published study suggested that the immunoScore signature could predict response to adjuvant chemotherapy in stage II and III GC patients in subgroup analysis, but the result was not validated (27). Another two studies reported that serum biomarkers based model in GC patients from single-center could classify the patients who are suitable for chemoradiation or chemotherapy $(28,29)$. However, these models were conducted in a small sample size and were not independently validated. The most extensive study in the prediction model derived from the CLASSIC study, which includes 746 patients in the discovery set and 943 in the validation set. However, it is only a survival predicting model with clinical variables (30). In the present study, we demonstrated a prediction model based on metabolic genes of chemo-reagents and clinic-pathological variables with high accuracy in the prediction of chemotherapy efficacy in two independent cohorts of LAGC patients. The discriminative capability of our model is comparable to other existing models (0.7-0.8), though the AUC in the TCGA cohort is relatively lower (0.67). It could be useful in the decision-making chemotherapy of LAGC.

The sensitivity to chemotherapy was suggested associating with the genomic and epigenomic landscape of GC. According to TCGA, GC was characterized into four molecular subtypes: Epstein-Barr virus (EBV)positive, micro-satellite instable (MSI), genomically stable (GS), and chrom instability (CIN). Among them, $A R I D 1 A$ was the most frequently identified mutated gene in EBV subtype, whereas TP53 and APC were the genes enriched in CIN subtype, PI3KCA, and $C D H 1$ were identified in MSI subtype and GS subtype (24), however, no specific drug sensitivity was tested in this study. Another Korean study indicated that GC with mesenchymal phenotype was resistant to standard chemotherapy (31). In our study, besides RRM1, DUT, TYMS, RRM2, TYMP, POLH, RRM2B and KI67, which were accessed in our two cohorts, the difference in chemo-sensitivity may be also associated with the mutation numbers and types in LAGC patients. The most commonly mutated gene in the high and medium chemo-signature group was TP53, and ARID1A mutations were mostly identified in low C-Score LAGC, which might be ascribed to CIN and EBV subtypes, respectively. Regarding the epithelial and mesenchymal phenotypes, C-Score was not associated with tumor grade in both cohorts $(P>0.05$, data not shown), but we did find that high tumor grade was higher in low C-Score LAGC compare to the median and high C-Score patients. Further, a high functional mutation rate identified in the low C-Score group indicated a high frequency of neo-antigens, 
which suggested that immunotherapy could be an ideal synergistic therapy with chemotherapy(32). Therefore, we proposed that C-Score was associated with molecular subtypes and immunotherapy of LAGC, though more research is warranted to decipher the underlying mechanisms.

Our study has the distinct advantages of relatively large sample size, two-phase design, validation in GC cell lines, and multi-variate predictive model. We acknowledge several limitations. First, we validated the mRNA level in the TCGA cohort, which was not consistent with the SRRSH cohort, which evaluated the protein level $(\mathrm{IHC})$ of the biomarkers. However, the principle of protein transcription and previous studies indicated the linear correlation between mRNA level and protein level (33), though epigenetic factors could not be ruled out (34). More independent cohorts using IHC detection for protein were required to validate our findings. Second, the heterogeneity between the two cohorts, like race, age, and gender, could not be alleviated, albeit the tumor stage, tumor grade and chemotherapy information were parallel. Third, the chemo-regimens of included LAGC were not unified, which may affect the results. However, all the regimens were based on oxaliplatin and 5-fluorouracil, which means the fundamental anti-tumor mechanisms were identical.

In summary, we identified and validated an eight gene signature associating with the responsiveness of chemotherapy in two independent cohorts of LAGC. Based on the signature and other clinical variables, we generated a risk model that could predict the survival of LAGC patients. Moreover, we also validated the results in a panel of GC cell lines. Mutation profiling based on the C-Score indicated that poor response to the chemotherapy in the low C-Score group might be associated with the number and type of mutations. The C-Score identified in this study could not only contribute to the stratification of the patients but also could help reduce the resistance to chemotherapy in LAGC.

\section{List Of Abbreviations}

GC: gastric cancer; LAGC: locally advanced gastric cancer; AC: Adjuvant chemotherapy; FFPE, formalinfixed and paraffin-embedded; RR, ribonucleotide reductase; IHC, immunohistochemistry; MTA, Multiple Tissue Array; OS: overall survival; TCGA: The Cancer Genome Atlas; SRRSH: Affilited Sir Run Run Shaw Hospital.

\section{Declarations}

Consent for publication:All authors agreed on the publication of this manuscript.

Disclosure of interest: The authors have no conflicts of interest to disclose.

\section{Funding}

This work wassupported by National Nature Science Foundation of China (No. 81972453, No. 81972597, No. 81602471 and No. 81672729), Nature Science Foundation of Zhejiang (Q16H160010, LY18H160005), Zhejiang Medical Scientific Research Foundation (2020RC022). 
Acknowledgement:We thank Mrs. Mariko Lee from the Microscope core Lab, City of Hope,for technical assistance with acquisition of immunohistochemistry microscopyimages; and Mrs. Yafan Wang from the Translational Medicine coreLab, City of Hope, for patients' information collection in the City of Hope set, and Ms. Yanyan Chai from the Oncology Surgery Dept, Sir RunRunShaw Hospital, for patients' information collection and follow-up in Zhejiang Universityset.

Author contributions: $\mathrm{QW}, \mathrm{XL}$ and $\mathrm{YY}$ designed the study. QW, CC and BX performed all data analysis. QW wrote the manuscript. $X \mathrm{~L}, \mathrm{LW}, \mathrm{CC}$ and $\mathrm{YY}$ revised the manuscript. All other authors contributed to the interpretation of data and approved the fnal manuscript.

\section{Data Availability Statement}

All data are available upon request after publication pending approval from the authors and subject to SRRSH rules and regulations regarding human subject research and material transfer agreement.

\section{References}

1. Bray F, Ferlay J, Soerjomataram I, Siegel RL, Torre LA, Jemal A. Global cancer statistics 2018 : GLOBOCAN estimates of incidence and mortality worldwide for 36 cancers in 185 countries. CA Cancer J Clin. 2018;68(6):394-424.

2. Isomoto H, Shikuwa S, Yamaguchi N, Fukuda E, Ikeda K, Nishiyama $H$, et al. Endoscopic submucosal dissection for early gastric cancer: a large-scale feasibility study. Gut. 2009;58(3):331-6.

3. Nakajima T, Nashimoto A, Kitamura M, Kito T, Iwanaga T, Okabayashi K, et al. Adjuvant mitomycin and fluorouracil followed by oral uracil plus tegafur in serosa-negative gastric cancer: a randomised trial. Gastric Cancer Surgical Study Group. Lancet. 1999;354(9175):273-7.

4. Neri B, de Leonardis V, Romano S, Andreoli F, Pernice LM, Bruno L, et al. Adjuvant chemotherapy after gastric resection in node-positive cancer patients: a multicentre randomised study. British journal of cancer. 1996;73(4):549-52.

5. D'Ugo D, Rausei S, Biondi A, Persiani R. Preoperative treatment and surgery in gastric cancer: friends or foes? Lancet Oncol. 2009;10(2):191-5.

6. Bang YJ, Kim YW, Yang HK, Chung HC, Park YK, Lee KH, et al. Adjuvant capecitabine and oxaliplatin for gastric cancer after D2 gastrectomy (CLASSIC): a phase 3 open-label, randomised controlled trial. Lancet. 2012;379(9813):315-21.

7. Sakuramoto S, Sasako M, Yamaguchi T, Kinoshita T, Fujii M, Nashimoto A, et al. Adjuvant chemotherapy for gastric cancer with S-1, an oral fluoropyrimidine. N Engl J Med. 2007;357(18):1810-20.

8. Sasako M, Sakuramoto S, Katai H, Kinoshita T, Furukawa H, Yamaguchi T, et al. Five-year outcomes of a randomized phase III trial comparing adjuvant chemotherapy with S-1 versus surgery alone in stage II or III gastric cancer. J Clin Oncol. 2011;29(33):4387-93. 
9. Cunningham D, Allum WH, Stenning SP, Thompson JN, Van de Velde CJ, Nicolson M, et al. Perioperative chemotherapy versus surgery alone for resectable gastroesophageal cancer. $\mathrm{N}$ Engl $\mathrm{J}$ Med. 2006;355(1):11-20.

10. Ychou M, Boige V, Pignon JP, Conroy T, Bouche O, Lebreton G, et al. Perioperative chemotherapy compared with surgery alone for resectable gastroesophageal adenocarcinoma: an FNCLCC and FFCD multicenter phase III trial. J Clin Oncol. 2011;29(13):1715-21.

11. Lee J, Lim DH, Kim S, Park SH, Park JO, Park YS, et al. Phase III trial comparing capecitabine plus cisplatin versus capecitabine plus cisplatin with concurrent capecitabine radiotherapy in completely resected gastric cancer with D2 lymph node dissection: the ARTIST trial. J Clin Oncol. 2012;30(3):268-73.

12. Ajani JA, D'Amico TA, Almhanna K, Bentrem DJ, Chao J, Das P, et al. Gastric Cancer, Version 3.2016, NCCN Clinical Practice Guidelines in Oncology. J Natl Compr Canc Netw. 2016;14(10):1286-312.

13. Park DJ, Lenz HJ. Determinants of chemosensitivity in gastric cancer. Curr Opin Pharmacol. 2006;6(4):337-44.

14. Kwon HC, Roh MS, Oh SY, Kim SH, Kim MC, Kim JS, et al. Prognostic value of expression of ERCC1, thymidylate synthase, and glutathione S-transferase P1 for 5-fluorouracil/oxaliplatin chemotherapy in advanced gastric cancer. Ann Oncol. 2007;18(3):504-9.

15. Wang Q, Liu X, Zhou J, Huang Y, Zhang S, Shen J, et al. Ribonucleotide reductase large subunit M1 predicts poor survival due to modulation of proliferative and invasive ability of gastric cancer. PLoS One. 2013;8(7):e70191.

16. Gao Y, Cui J, Xi H, Cai A, Shen W, Li J, et al. Association of thymidylate synthase expression and clinical outcomes of gastric cancer patients treated with fluoropyrimidine-based chemotherapy: a meta-analysis. Onco Targets Ther. 2016;9:1339-50.

17. Huang L, Liu S, Lei Y, Wang K, Xu M, Chen Y, et al. Systemic immune-inflammation index, thymidine phosphorylase and survival of localized gastric cancer patients after curative resection. Oncotarget. 2016.

18. Metzger R, Leichman CG, Danenberg KD, Danenberg PV, Lenz HJ, Hayashi K, et al. ERCC1 mRNA levels complement thymidylate synthase mRNA levels in predicting response and survival for gastric cancer patients receiving combination cisplatin and fluorouracil chemotherapy. J Clin Oncol. 1998;16(1):309-16.

19. Ichikawa W, Takahashi T, Suto K, Shirota Y, Nihei Z, Shimizu M, et al. Simple combinations of 5-FU pathway genes predict the outcome of metastatic gastric cancer patients treated by S-1. Int J Cancer. 2006;119(8):1927-33.

20. Tomicic MT, Aasland D, Naumann SC, Meise R, Barckhausen C, Kaina B, et al. Translesion polymerase eta is upregulated by cancer therapeutics and confers anticancer drug resistance. Cancer Res. 2014;74(19):5585-96.

21. Kim HK, Choi IJ, Kim CG, Kim HS, Oshima A, Michalowski A, et al. A gene expression signature of acquired chemoresistance to cisplatin and fluorouracil combination chemotherapy in gastric cancer 
patients. PLoS One. 2011;6(2):e16694.

22. Lei Z, Tan IB, Das K, Deng N, Zouridis H, Pattison S, et al. Identification of molecular subtypes of gastric cancer with different responses to PI3-kinase inhibitors and 5-fluorouracil. Gastroenterology. 2013;145(3):554-65.

23. Longley DB, Harkin DP, Johnston PG. 5-fluorouracil: mechanisms of action and clinical strategies. Nat Rev Cancer. 2003;3(5):330-8.

24. Cancer Genome Atlas Research N. Comprehensive molecular characterization of gastric adenocarcinoma. Nature. 2014;513(7517):202-9.

25. Liu X, Zhang H, Lai L, Wang X, Loera S, Xue L, et al. Ribonucleotide reductase small subunit M2 serves as a prognostic biomarker and predicts poor survival of colorectal cancers. Clin Sci (Lond). 2013;124(9):567-78.

26. Zhou B, Phan V, Liu X, Juhasz A, Chu PG, Yen Y. Production of a monoclonal antibody against the hRRM2 subunit of ribonucleotide reductase and immunohistochemistry study of human cancer tissues. Hybridoma. 2006;25(5):264-70.

27. Jiang Y, Zhang Q, Hu Y, Li T, Yu J, Zhao L, et al. ImmunoScore Signature: A Prognostic and Predictive Tool in Gastric Cancer. Ann Surg. 2018;267(3):504-13.

28. Qin R, Yang Y, Chen H, Qin W, Han J, Gu Y, et al. Prediction of neoadjuvant chemotherapeutic efficacy in patients with locally advanced gastric cancer by serum IgG glycomics profiling. Clin Proteomics. 2020;17:4.

29. Ahn HS, Sohn TS, Kim MJ, Cho BK, Kim SM, Kim ST, et al. SEPROGADIC - serum protein-based gastric cancer prediction model for prognosis and selection of proper adjuvant therapy. Sci Rep. 2018;8(1):16892.

30. Jiang Y, Li T, Liang X, Hu Y, Huang L, Liao Z, et al. Association of Adjuvant Chemotherapy With Survival in Patients With Stage II or III Gastric Cancer. JAMA Surg. 2017;152(7):e171087.

31. Oh SC, Sohn BH, Cheong JH, Kim SB, Lee JE, Park KC, et al. Clinical and genomic landscape of gastric cancer with a mesenchymal phenotype. Nat Commun. 2018;9(1):1777.

32. Samstein RM, Lee CH, Shoushtari AN, Hellmann MD, Shen R, Janjigian YY, et al. Tumor mutational load predicts survival after immunotherapy across multiple cancer types. Nat Genet. 2019;51(2):202-6.

33. Chen YT, Hsu M, Lee P, Shin SJ, Mhawech-Fauceglia P, Odunsi K, et al. Cancer/testis antigen CT45: analysis of mRNA and protein expression in human cancer. Int J Cancer. 2009;124(12):2893-8.

34. Harvey ZH, Chen Y, Jarosz DF. Protein-Based Inheritance: Epigenetics beyond the Chromosome. Mol Cell. 2018;69(2):195-202.

\section{Figures}



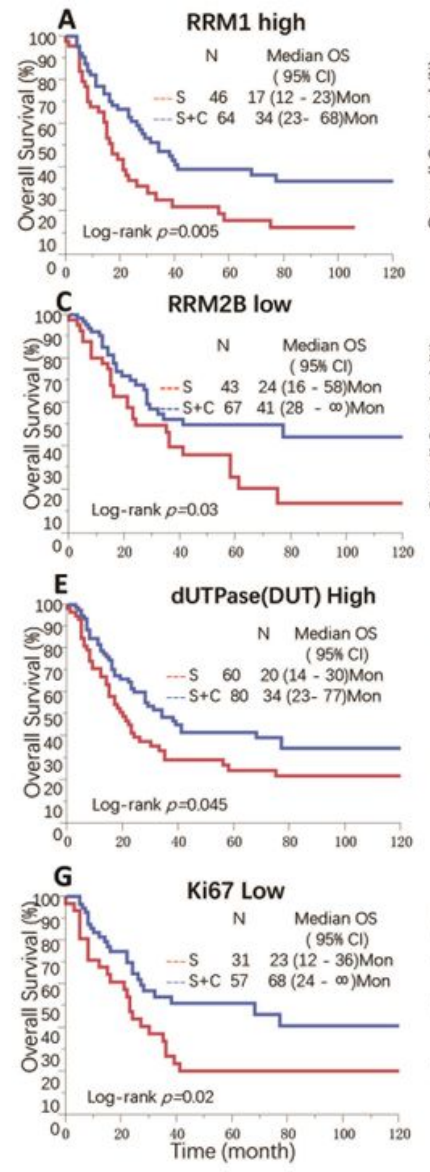

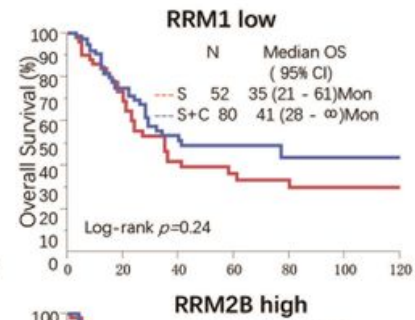

$\mathrm{N} \quad$ Median os

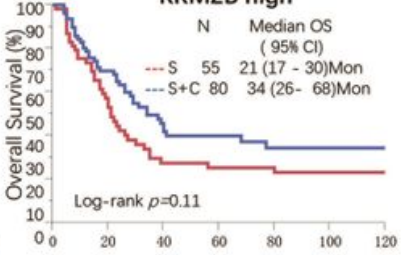

dUTPase(DUT) low
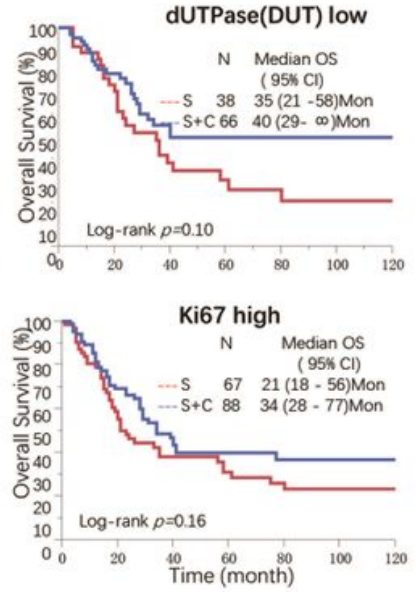
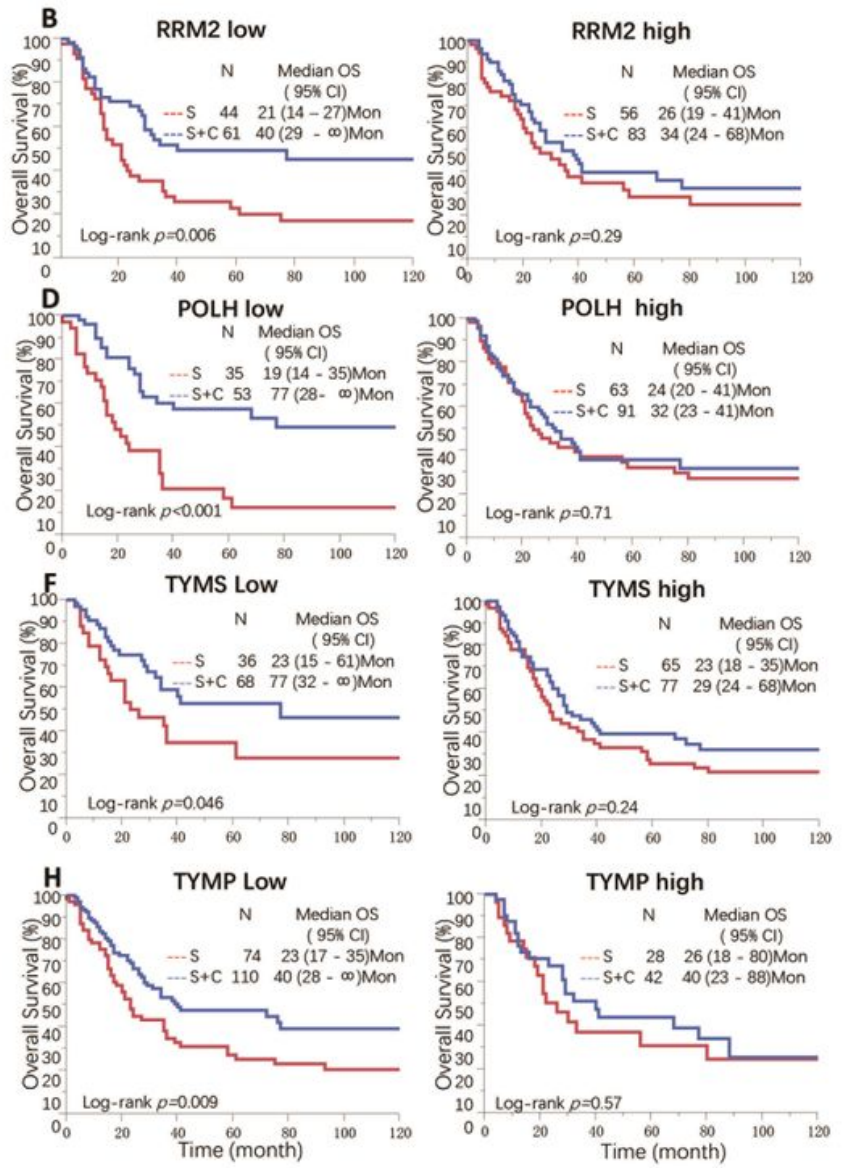

\section{Figure 1}

C-Score biomarkers predict the efficacy of chemotherapy in LAGC in both SRRSH and TCGA cohorts CScore biomarkers were evaluated in both SRRSH and TCGA cohorts, Kaplan-Meier analysis indicated that high RRM1 (A),low RRM2(B), low RRM2B(C), low POLH(D), high DUT (E), low TYMS(F), low KI67(G) or low $\operatorname{TYMP}(\mathrm{H})$ were associated with better response to adjuvant chemotherapy compare to low RRM1, high RRM2, high RRM2B, high POLH, low DUT, high TYMS, high KI67 or high TYMP, respectively. 

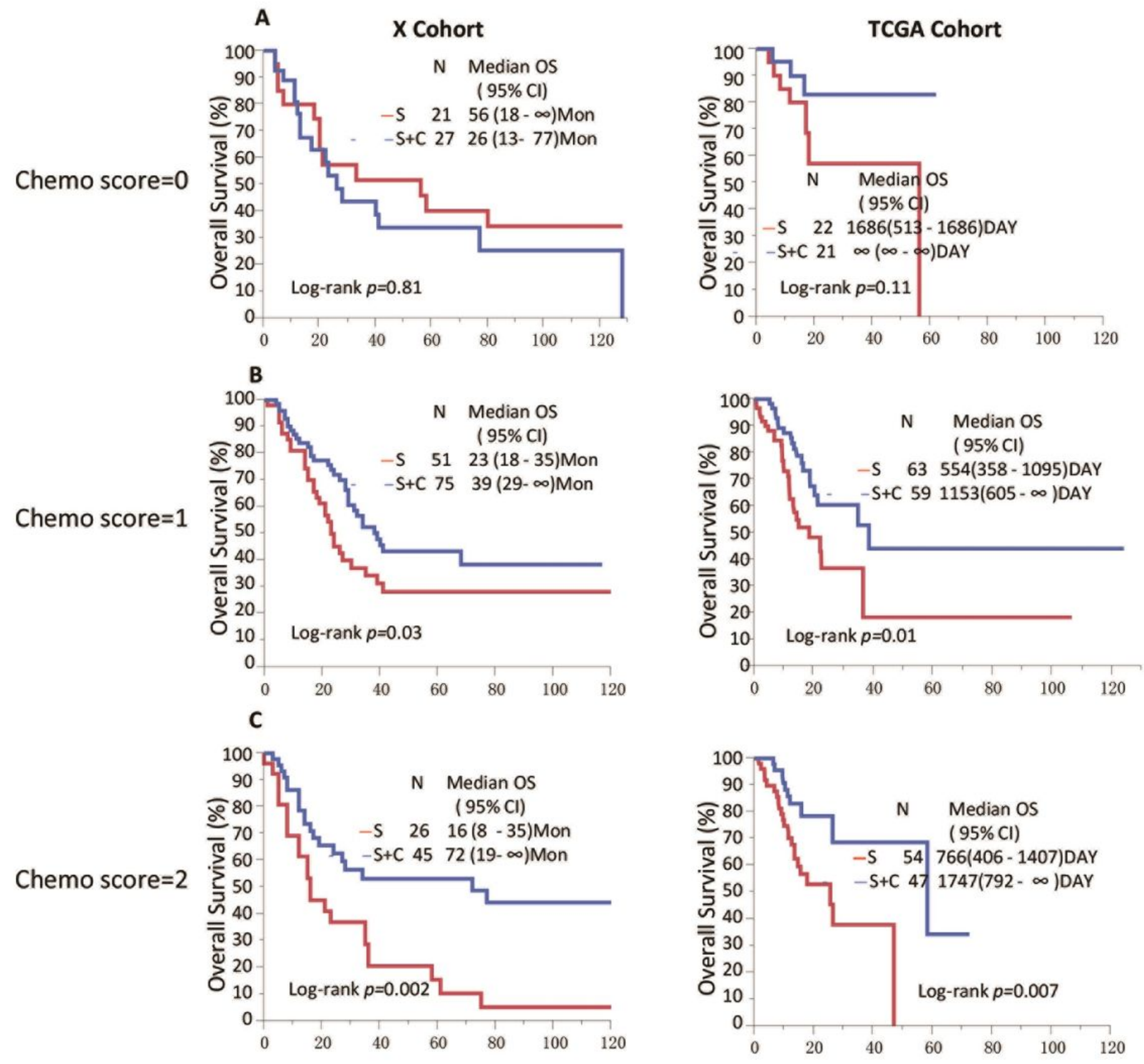

Figure 2

C-Scoreis an ideal predictor for adjuvant therapy efficacy in LAGC A. In LAGC patients with low C-Score, adjuvant chemotherapy demonstrated poor efficacy in prolong OS (log-rank $p=0.81$ and 0.11 in SRRSH and TCGA cohort, respectively). B. In LAGC patients with medium chemo-score, adjuvant chemotherapy showed significant improved OS compare to surgery only patients (log-rank $p=0.03$ and 0.01 in SRRSH and TCGA cohort, respectively). C.In LAGC patients with highC-Score, adjuvant chemotherapy showed significant improved OS compare to surgery only patients (log-rank $p=0.002$ and 0.007 in SRRSH and TCGA cohort, respectively) 
A

\section{$x$ Cohort}
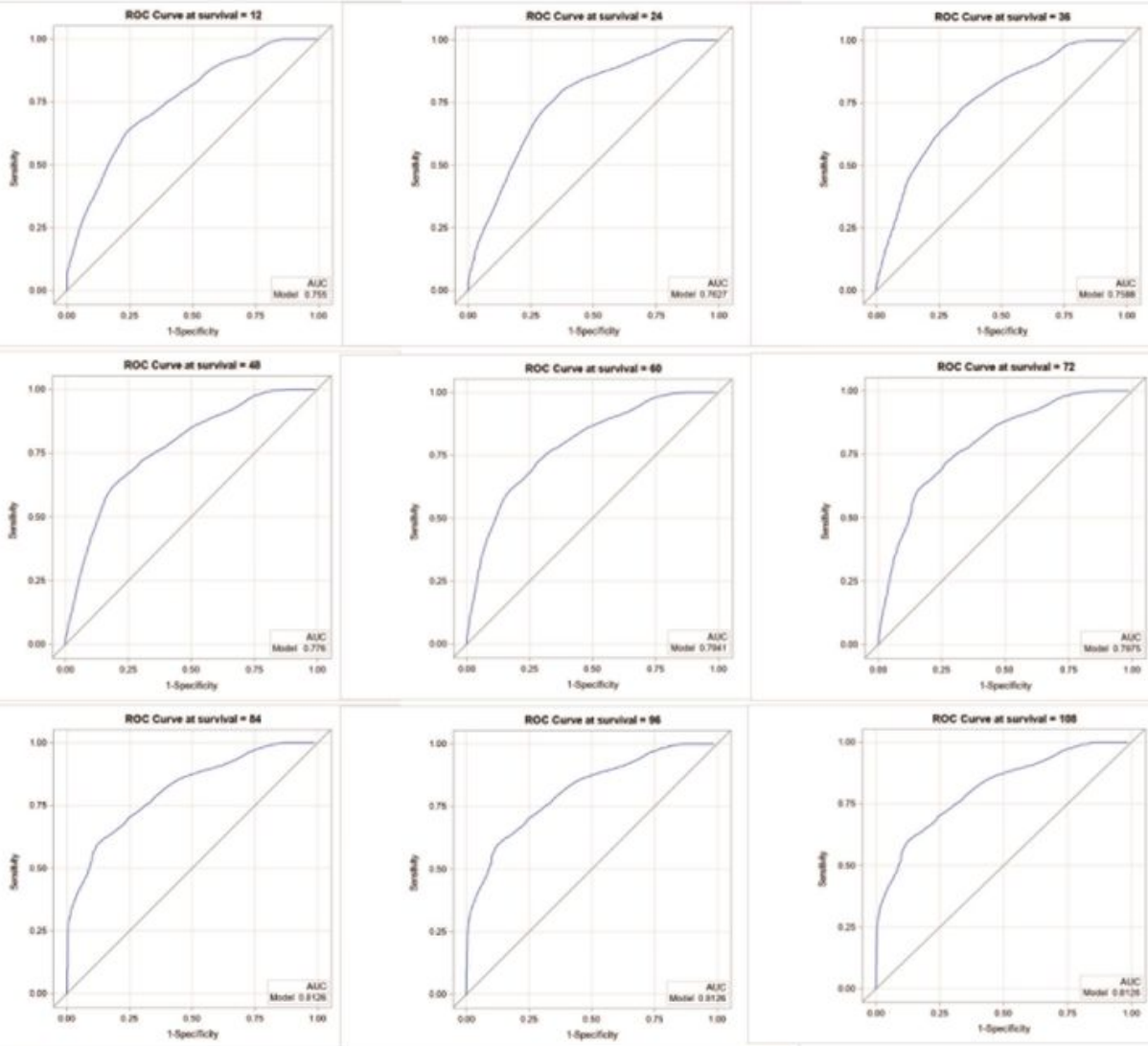

B

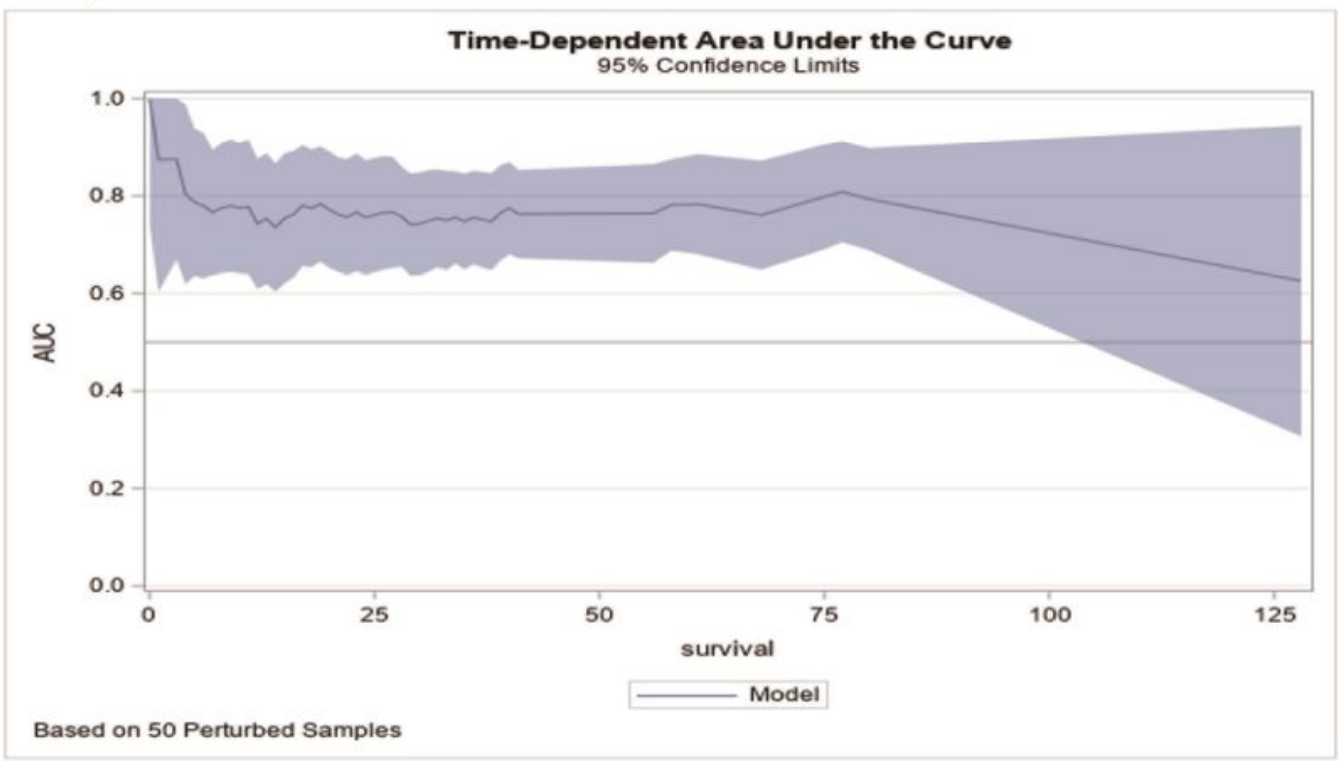

\section{Figure 3}

Prediction model based on C-Score could successfully predict efficacy of adjuvant chemotherapy in LAGC A.The ROC curves and AUC of the model, AUC values fluctuated between 0.75 to 0.81 across different survival tie points, with the highest AUC in 84, 96 and 108 months (0.8126). B.The timedependent AUC and corresponding 95\% confidence limits were presented 
A
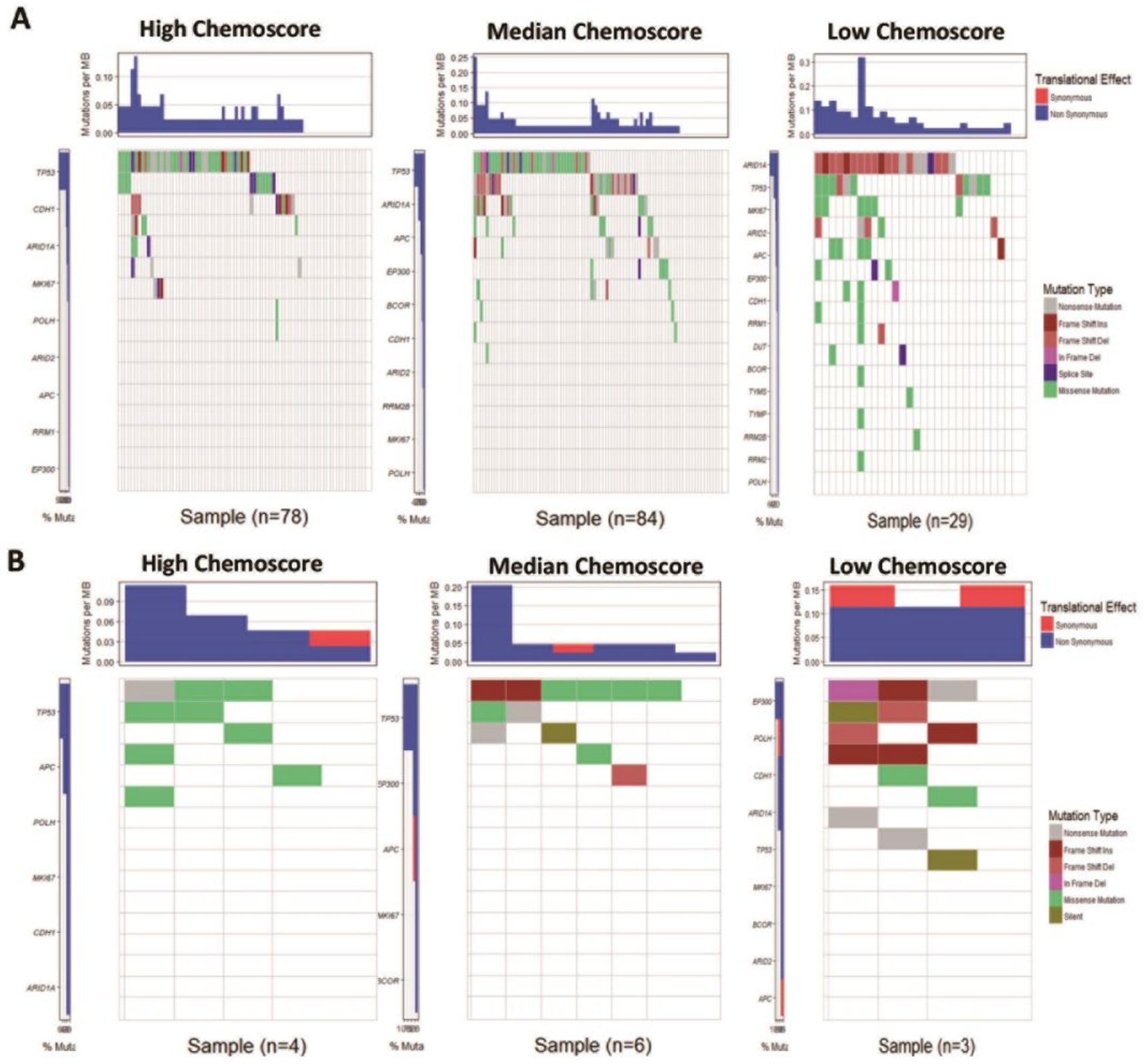

\section{Figure 4}

TheC-Score is associated with mutation type and burden in LAGC A.The waterfall plots of mutation profiles of LAGCs from TCGA were depicted, the patients with low C-Score demonstrated a significantly higher rate of functional mutations than group median and high group. B. The waterfall plots of mutation profiles of 28 gastric cancer celllines. Celllines with low chemo-signature (group low) showed high frequency of frameshift mutations in and high frequency of missense mutation than group median and group high celllines.

\section{Supplementary Files}


This is a list of supplementary files associated with this preprint. Click to download.

- Supplementalmaterials.docx

- Supplementalmaterials.docx 